\title{
EFEITO DO FUNDO CONSTITUCIONAL DO CENTRO-OESTE NA GERAÇÃO DE EMPREGOS
}

\author{
EFFECT OF CONSTITUTIONAL FOUND OF THE BRAZILIAN MIDWEST IN THE \\ GENERATION OF EMPLOYMENT
}

\author{
Carlos Eduardo de Freitas ${ }^{1}$ \\ João Bosco Arbues Carneiro Junior ${ }^{2}$ \\ Amauri do Prado Justino
}

\begin{abstract}
RESUMO
O Fundo Constitucional do Centro-Oeste (FCO) visa reduzir as desigualdades regionais disponibilizando recursos ao setor privado através de projetos de investimento. Os relatórios de gestão não promovem uma avaliação substantiva dos resultados. Nesse contexto buscou-se verificar a efetividade na geração de empregos e aos objetivos socioeconômicos através da tipologia descritiva, sob uma abordagem quali-quantitativa. Dos dados extraídos entre os 468 municípios pesquisados da região Centro-Oeste nos anos 1985 e 1996 (antes e depois da implantação do programa). Os resultados apontaram uma geração média de empregos para o comércio de 5.493 empregos, indústria 1.331 e o setor de serviços de 3.559. Isso equivale a $28 \%$ dos empregos gerados no período em todos os setores e a $65 \%$ das vagas estimadas pelo fundo na liberação dos recursos. A importância do FCO fica demonstrada ao identificar o crescimento do PIB regional e setorial e pelo número crescente de formalização de empresas.
\end{abstract}

Palavras-chave: Desigualdades regionais; Políticas Regionais de Desenvolvimento; Fundo Constitucional do Centro-Oeste.

\begin{abstract}
The Constitutional Fund of the Midwest (FCO) aims to reduce regional inequalities by providing resources to the private sector through investment projects. management reports do not promote a substantive evaluation of the results. In this context we sought to assess the effectiveness in job creation and socio-economic objectives through descriptive type, under a qualitative and quantitative approach. Data extracted from the 468 municipalities surveyed in the Midwest region in the years 1985 and 1996 (before and after program implementation). The results showed an average job creation for

\footnotetext{
${ }^{1}$ Graduado em Economia pela Universidade Estadual de Maringá (UEM). Mestre em Teoria Econômica pela Universidade Estadual de Maringá (UEM).Doutor em Economia Aplicada pela Universidade Federal de Pernambuco (UFPE). Professor da Universidade Federal de Mato Grosso (UFMT).

Emial:cfreitas@ufmt.br

${ }^{2}$ Graduado em Ciências Contábeis pela Universidade Federal de Mato Grosso (UFMT). Especialista em Administração Contábil-Financeira pela Universidade Federal de Mato Grosso (UFMT). Mestre em Ciências Contábeis pela Universidade Federal do Rio de Janeiro (UFRJ). E-mail: jbacj@hotmail.com
} 
trading 5,493 jobs, 1,331 industry and 3,559 in the service sector. This equates to $28 \%$ of the jobs generated in the period in all sectors and $65 \%$ of vacancies estimated by the fund in the release of funds. The importance of the FCO is demonstrated by identifying the growth of regional and sectoral GDP and the growing number of formal companies.

Keywords: Regional Inequalities; Policies Regional Development; Constitutional Fund of the Midwest.

JEL: O18; R11; R58.

\section{Introdução}

Por suas características particulares em termos de complexidade e diversidade, $\mathrm{o}$ Brasil tem desafios e resultados significantes no que tange a formulação de políticas de desenvolvimento regional e social. Uma medida governamental foi à criação do FCO, regulamentado como política de desenvolvimento e tem a finalidade de garantir recursos financeiros para os setores produtivos da região Centro-Oeste buscando promover o equilíbrio socioeconômico regional.

De acordo com Brum (1984) "as disparidades regionais de riqueza e de renda não são um fenômeno novo na vida brasileira, sendo, um dos resultados mais graves do processo histórico pelo qual se desenvolveu a economia brasileira (BRUM 1984 apud SILVA \& BUESCU, 1974, p.68)”. As cidades foram crescendo rapidamente sem infraestrutura e planejamento adequado, gerando consequências sociais para o país; o processo de desenvolvimento e crescimento brasileiro possui um contexto histórico onde a economia se expande aceleradamente a partir do século XX ocorrendo grande concentração de renda e índice populacional na região Sul/Sudeste (ALCOFORADO, 2003; PEREIRA e PÔRTO JÚNIOR, 2001).

A partir da década de 1950 o governo federal passou a adotar políticas regionais de desenvolvimento, buscando amenizar as disparidades sociais decorrentes da concentração econômica de investimentos e infraestrutura na região Sudeste e Sul e integrar as demais regiões do país para expandir a economia brasileira; e sentindo a necessidade de aperfeiçoamento e garantia de recursos para esses programas foram criados os Fundos Constitucionais de Financiamento.

Em estudo de 2010 do Programa das Nações Unidas para o Desenvolvimento (PNUD) sobre a América Latina e no Caribe, o Brasil aparece na terceira colocação de pior índice de desigualdade social do mundo. Luna e Klein (2009) destacam que a 
desigualdade social no Brasil possui índices elevados colocando o país no ápice das discussões mundiais sobre o tema.

Estudo do Banco Mundial em 1988 aponta que aproximadamente um terço dos brasileiros viviam em situação de pobreza e destaca a existência de desigualdade social. Desigualdades sociais são evidenciadas principalmente nas regiões Norte, Nordeste e Centro-Oeste (ALCOFORADO, 2003; LUNA e KLEIN, 2009; COLON, 2010), consideradas regiões menos desenvolvidas que por sua vez apresentam índices sociais que não se equiparam aos índices das regiões Sul e Sudeste do país. Estes vêm diminuindo, porém permanecem, e os desequilíbrios regionais afetam não somente as regiões Norte, Nordeste e Centro-Oeste, mas o desenvolvimento do país como um todo (LAMPREIA, 1995).

Com a mesma visão, Moreno \& Higa (2005) afirmam que "mesmo com várias políticas adotadas a problemática desigualdade social permanece”. Porém, esta apresenta redução devido aos investimentos públicos contínuos na região, os índices sociais da região Centro-Oeste tiveram uma melhora absoluta na década de 1970, evidenciados pelas políticas governamentais, como os incentivos fiscais e financiamentos.

Na década de 1970 estudos entre as regiões brasileiras já apontavam as disparidades sociais do país. Segundo Lampreia (1995), em 1970 a distribuição populacional pelo território era altamente diferenciada entre às regiões do país, a região Norte $45,26 \%$ do território nacional possuía 5,90\% da população, na região Sudeste $10,85 \%$ do território e $43,5 \%$ da população. Na região Nordeste $18,27 \%$ da área total e $28,5 \%$ da população, e na região Centro-Oeste $18,86 \%$ da área total e $6,80 \%$ da população, denotando a discrepância na densidade demográfica.

A região Centro-Oeste é celeiro de várias políticas públicas, incentivos fiscais e financeiros. Segundo Monteiro Neto \& Gomes (1999, p. 856) “[...] a participação do Governo na economia tem ultrapassado os 50\% do PIB regional" e complementa que “...o papel do setor público tem sido de fundamental importância na explicação do alto desempenho do PIB per capita". A partir dos investimentos públicos as regiões podem melhorar seus índices sociais e diminuir as desigualdades regionais. O investimento público coloca no mercado recursos antes inexistentes e movimenta a economia local.

O FCO coloca a disposição da região Centro-Oeste recursos a médio e longo prazo, com taxas de juros próximas da inflação, prazos de pagamentos e de carência 
dilatados, tornando-se fonte de financiamento diferenciado no mercado de créditos. Para a obtenção destas linhas de financiamento é necessário seguir uma série de exigências primordiais para a seguridade de direitos e responsabilidades, de acordo com o porte e necessidades esclarecidas pela organização; iniciando pelo projeto de investimento (CARTILHA FCO, 2001).

Em análise dos relatórios de gestão do FCO depara-se com a deficiência de informações sobre a efetividade do fundo. Apenas são demonstradas projeções do número de empregos gerados e valores financiados. Há falha na avaliação, efetividade e resultados alcançados pelo FCO. Segundo Rollemberg (2013, p.7) em seu parecer sobre o relatório do FCO encaminhado ao Senado Federal "as informações indicam, essencialmente, o número de operações e o valor do crédito concedido. Não há referências ao padrão de atividades produtivas ou às especificidades do plano a cada sub-região em particular". Ainda complementa, que o parecer não avalia o impacto do FCO segundo as prioridades estabelecidas no Plano Estratégico de Desenvolvimento do Centro-Oeste (ROLLEMBERG, 2013).

Estes relatórios de gestão deveriam conter informações completas sobre a aplicação dos recursos e os resultados alcançados conforme o plano estratégico para o desenvolvimento da região e microrregiões. Dessa forma, para verificar a contribuição da política regional com o desenvolvimento da região Centro-Oeste foram analisadas variáveis antes e após a criação do FCO (1985/1996) e questiona-se: Qual a relação entre os recursos liberados pelo FCO e a geração de empregos?

O objetivo geral do estudo consiste em analisar a relação entre a aplicação dos recursos do FCO e a geração de empregos. Para atingir este definiram-se os seguintes objetivos específicos: (a) Demonstrar a importância do FCO para a região Centro-Oeste; (b) Construir um modelo econométrico para verificar se o montante de recursos liberados explica a quantidade de empregos gerados; e (c) Aplicar testes de inferências para verificar a validação do modelo criado.

Apresentam-se como hipóteses iniciais ao problema abordado, $\mathrm{H}_{0}$ - acredita-se que a liberação de recursos do fundo não influencie o número de empregos gerados e $\mathrm{H}_{1}$ - há relação entre a aplicação dos recursos do FCO e o número de empregos gerados.

O estudo justifica-se pela necessidade de evidenciação de resultados sociais e regionais, já que os relatórios de gestão não contemplam informações gerenciais de 
aplicação. Também, a relevância está na importância das políticas de desenvolvimento e de integração nacional a qual é necessária analisar a eficácia da aplicação dos recursos, bem como verificar se o objetivo de diminuir as desigualdades regionais e de integração nacional a que o fundo foi criado está sendo alcançado.

\section{REVISÃO DE LITERATURA}

\subsection{Fundo Constitucional do Centro-Oeste}

A origem do FCO foi através da CF de 1988, art. 159, inciso I, alínea "c" regulamentado pela Lei $\mathrm{n}^{\mathrm{o}}$ 7.827, de 27 de setembro de 1989, onde se criaram os Fundos Constitucionais de Financiamento, objetivando promover o equilíbrio socioeconômico entre as regiões do país.

Os Fundos Constitucionais homologados para acelerar o desenvolvimento nas regiões mais carentes do Brasil, são: FNE - Fundo Constitucional do Nordeste; FNO Fundo Constitucional do Norte; e FCO - Fundo Constitucional do Centro-Oeste. As regiões de atuação de cada fundo são claramente identificadas no mapa abaixo:

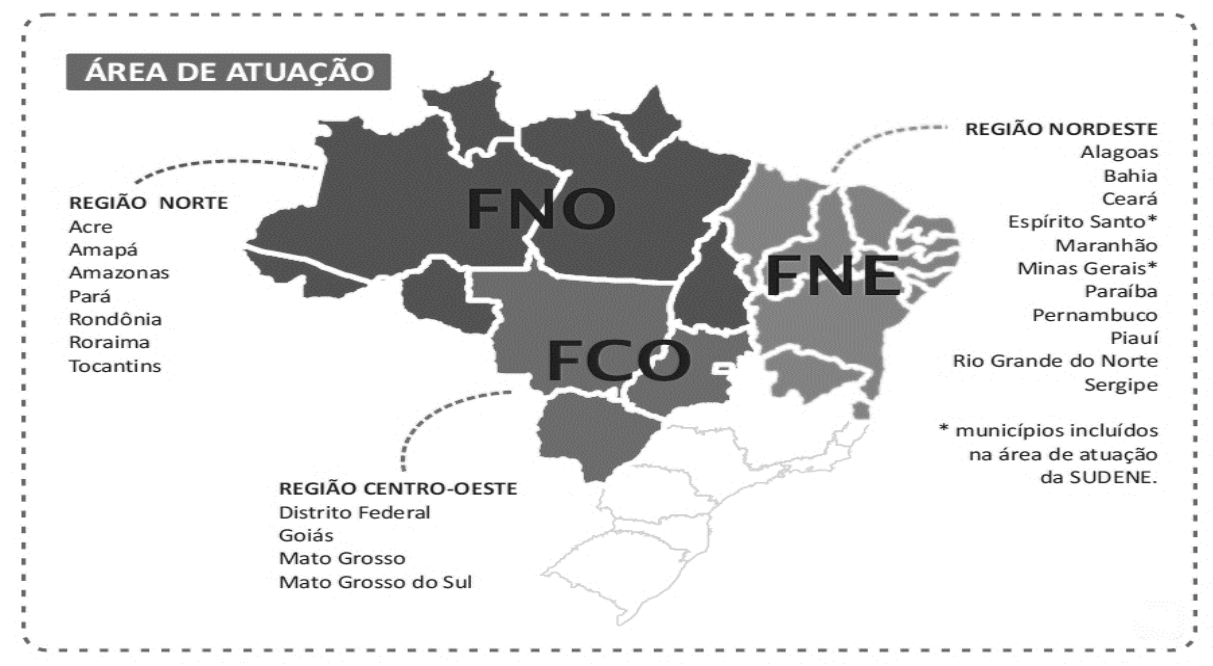

Figura 1 - Descrição das regiões e área de atuação de cada Fundo.

Fonte: Sistemas de informações gerenciais - Ministério da integração Nacional, 2011.

A CF (1988), inciso 159 determina a destinação de 3\% do produto da arrecadação dos impostos sobre renda, proventos de qualquer natureza e sobre produtos industrializados para aplicação em programas de financiamento aos setores produtivos destas regiões. A distribuição dos recursos entre as regiões corresponde à determinação da $\mathrm{CF}$, o qual prioriza a região Nordeste e o semiárido, ficando a seguinte distribuição 
de $1,8 \%$ ao FNE, 0,6\% ao FNO e 0,6\% ao FCO (Lei N 7.827 , de 27 de Setembro de 1989).

Desde a criação dos Fundos em 1989 os recursos foram aplicados em programas de financiamento dos setores produtivos (agropecuário, mineral, agroindustrial, industrial e turismo) e a partir de 2003, após ajustes advindos da Lei $\mathrm{n}^{\mathrm{o}} 10.177$ de Janeiro de 2001 abriu-se crédito aos setores de infraestrutura, comércio e serviços.

A Cartilha do FCO (2001) traz que:

[...] os programas de financiamento buscam maior eficácia na aplicação dos recursos, de modo a aumentar a produtividade dos empreendimentos, gerar novos postos de trabalho, aumentar a arrecadação tributária e melhorar a distribuição da renda. $\mathrm{O}$ direcionamento de recursos aos investimentos de longo prazo permite que os projetos assistidos contribuam para o desenvolvimento regional sustentável e promovam a modernização das atividades econômicas tradicionais, com melhoria de competitividade e sustentabilidade dos agentes de produção (BRASIL, p.5, 2001).

A missão do fundo é a redução das desigualdades sociais entre as regiões e contribuir para o desenvolvimento da região e do país como um todo.

Conforme as cartilhas disponibilizadas a cada ano, toda programação está segmentada por setores produtivos (empresarial e rural), sendo os recursos aplicados no âmbito dos seguintes programas.

\section{PROGRAMAS DE FINANCIAMENTO - FCO}

FCO RURAL

Linhas de Crédito de:

Desenvolvimento Rural;

Desenvolvimento de Irrigação e Drenagem;

Desenvolvimento de Sistema de Integração Rural (CONVIR);

Integração de Lavoura-Pecuária

Conservação da Natureza;

- Retenção de Matrizes na Planície Pantaneira;

Apoio ao Desenvolvimento da Aqüicultura;

Apoio ao Desenvolvimento da Pesca;

Linha Especial de Crédito para Adequaca ado Sistemade

Produção Pecuário na Região de Fronteira;

Linha Especial de Financiamento para Custeio

Agropecuário para Médios e Grandes Produtores Rurais.

PROGRAMA NACIONAL DE FORTALECIMENTO DA AGRICULTURA FAMILIAR - PRONAF

PROGRAMA NACIONAL DE FORTALECIMENTO DA AGRICULTURA FAMILIAR - PRONAF - REFORMA AGRÁRIA (PLANTABRASIL)
FCO EMPRESARIAL DE APOIO AOS MICROEMPREENDEDORES INDIVIDUAIS ÀS MICRO E PEQUENAS EMPRESAS

Linhas de Crédito de:

Desenvolvimento Industrial para MEI e MPE;

Desenvolvimento do Turismo Regional para MEl e MPE,

Desenvolvimento dos Setores Comercial e de Serviços para MEI e MPE.

FCO EMPRESARIAL PARA MÉDIAS E GRANDES EMPRESAS

Linhas de Crédito de:

Desenvolvimento Industrial para MGE;

Econômica para MGE;

Desenvolvimento do Turismo Regional para MGE;

Desenvolvimento dos Setores Comercial e de Serviços para MGE.

FCO EMPRESARIAL PARA REPASSES

FCO RURAL PARA REPASSES

Figura 2 - Descrição dos programas de financiamento do FCO.

Fonte: Sistemas de informações gerenciais - Ministério da integração Nacional, 2011. 
Observam-se na Figura 2 os programas atendidos pelo FCO a) Programa de FCO Empresarial de Apoio aos Empreendedores Individuais - EI e às Micro e Pequenas Empresas - MPE; b) Programa de FCO Empresarial para Pequena-Médias, Médias e Grandes Empresas - MGE; c) Programa de FCO Rural; d) Programa Nacional de Fortalecimento da Agricultura Familiar - Pronaf; e) Programa de FCO Empresarial para Repasse; e f) Programa de FCO Rural para Repasse (BRASIL, 2012, p.04). A divisão dos programas permite uma análise mais aprofundada e facilita quando o objetivo é destinar mais recursos a um setor, e analisar seus resultados.

Os Fundos Constitucionais de Financiamento visam corrigir desequilíbrios regionais, a fim de garantir crescimento e melhoria das condições socioeconômicas. Todo o processo dirigido pelas diretrizes, metas e programas estabelecidos visam à eficácia na aplicação dos recursos.

Para a obtenção destas linhas de financiamento é necessário seguir uma série de exigências primordiais para a seguridade de direitos e responsabilidades, de acordo com o porte e necessidades esclarecidas pela organização. Estas exigências devem constar no projeto de investimento.

\subsection{Projetos de investimento}

O FCO é um fundo de investimento do setor público, e para alcance destes recursos os empresários devem iniciar pelo projeto de investimento. Buarque (1991 apud Caldas 1984 p. 25) define projetos de investimento como "um conjunto ordenado de antecedentes, pesquisas, suposições e conclusões, que permitem avaliar a conveniência (ou não) de destinar fatores e recursos para o estabelecimento de uma unidade de produção determinada". Trata-se de um processo de análise da viabilidade ou não do investimento.

Buarque (1991, p.132) apresenta que os organismos de fomento foram criados com base na constatação de que um dos problemas fundamentais do processo de desenvolvimento, principalmente industrial, era a escassez de capital nos países subdesenvolvidos. Para resolver esse problema, o governo, por meio das agências financeiras especializadas no fomento de novas unidades de produção poderiam 
fornecer os recursos necessários ao setor privado para a geração de novas unidades de produção ou para a ampliação das já existentes.

As avaliações de projetos realizadas com a finalidade da obtenção de fundos diferenciam-se do setor privado, pois, além da rentabilidade, os projetos devem atender a objetivos nacionais. Para o FCO, o proponente deve preencher requisitos e exigências que constam no formulário modelo fornecido pelo Banco do Brasil. Neste devem ser informados todos os dados da empresa e estimativas de geração de empregos, uso de tecnologias avançadas, matéria-prima nacional, melhoria da renda e produtividade (BANCO DO BRASIL, 2015). Ou seja, de que forma será a geração de benefícios regionais e socioeconômicos.

Buarque (1991) afirma que, para determinar se a unidade de produção estudada por meio do projeto é justificável, os interessados devem realizar avaliação do projeto e se este representa uma boa alternativa para os recursos a serem utilizados. Falcini (1995) afirma que avaliar significa determinar a valia ou valor de alguma coisa; estimar significa determinar, por cálculo ou avaliação, o preço, o valor ou a valia de alguma coisa; avaliação ou estimativa tem, portanto, o mesmo significado na determinação de valor.

O projeto de investimento é o meio utilizado para a obtenção de recursos do FCO, este contém informações sobre a empresa, pesquisas comerciais, projeções de rentabilidade e socioeconômicas. É através do projeto de investimento que o banco regional analisa a viabilidade ou não do investimento frente aos objetivos sociais e regionais do fundo.

\subsection{Desenvolvimento Regional}

O processo de desenvolvimento regional deu-se com o esforço histórico da intervenção do Estado nas atividades setoriais. Desde a criação de Planos Nacionais de Desenvolvimento, a marcha para o oeste, incentivos fiscais e renúncias tributárias. $\mathrm{O}$ Estado sempre atuou na definição de marcos regulatórios, fomento à iniciativa privada e também como agente econômico direto de algumas atividades produtivas.

$\mathrm{Na}$ definição de Bresser (2007), “o desenvolvimento econômico é possível somente quando um estado-nação pode contar com um estado eficaz". O estado brasileiro busca a eficácia direcionando recursos financeiros ao desenvolvimento 
regional, no sentido oportunizar aportes financeiros a investidores locais e estes produzirem a redução de desigualdades sociais.

SILVA (2005) ressalta que "eficiência significa fazer acontecer com racionalidade o que implica medir os custos que a satisfação das necessidades públicas importa em relação ao grau de utilidade alcançado". A avaliação é feita através da mensuração dos custos e resultados. A eficácia do Estado na gestão de políticas públicas é medida através do controle na administração pública, mensurando os investimentos e a redução das desigualdades sociais e regionais.

Exemplifica-se como políticas de desenvolvimento a criação da Superintendência do Desenvolvimento da Região Centro-Oeste (SUDECO) em 1967, a criação dos programas especiais como Programa de Integração Nacional, POLOAMAZONIA, POLOCENTRO, PROÁLCOOL entre outros.

Os fundos constitucionais (FCO, FNE e FNE) propiciam mobilização por projetos de desenvolvimento regional flexíveis, capaz de promover o desenvolvimento regional e atender os agentes locais de desenvolvimento devido à escassez na oferta de recursos. As políticas adotadas destinaram-se como instrumentos de políticas públicas, investindo em setores prioritários ao desenvolvimento econômico das regiões visando dinamizar a economia regional, gerar empregos, incrementar a renda e ofertar produtos e serviços a população.

De acordo com Bevilacqua (2010, p.13) atualmente a intervenção do Estado condiz com direcionamentos das principais forças econômicas do mundo, destaca-se o grupo BRICS (Brasil, Rússia, Índia, China e África do Sul) por elevar a discussão acerca da intervenção do Estado, extrapolando a pauta de exportações e incluindo proteção do meio ambiente, integração regional, equilíbrio fiscal, defesa comercial e subvenções agrícolas. A maximização da pauta do BRICS demonstra preocupação com o desenvolvimento sustentável.

Em aspectos de políticas de subsídio para o desenvolvimento regional, o Estado brasileiro tem uma participação pequena frente aos demais países em desenvolvimento. O estudo da Organização Mundial do Comércio (OMC) - Word Trade Report (2006), dentre os países em desenvolvimento, o Brasil situa-se como um dos que menos concedem subsídios ao setor privado, representam $0,3 \%$ do PIB e corresponde a $1,8 \%$ das despesas públicas federais. 
As políticas de incentivo desenvolvidas nas regiões, estados e munícipios brasileiros é complexa, pois as diversidades e distanciamento geográfico podem ter contribuído para tal situação. A revisão e a discussão do papel do Estado geram posições bastante divergentes, porém o papel do Estado é fundamental para a redução da desigualdade social.

\section{PROCEDIMENTOS METODOLÓGICOS}

A metodologia constitui os procedimentos tomados para resolução da problemática encontrada, a trajetória metodológica que se seguiu para a concretização desta pesquisa foi definida através do enquadramento metodológico, população, amostra pesquisada, procedimentos e coleta dos dados.

$\mathrm{Na}$ pesquisa foram analisados os índices sociais da região Centro-Oeste disponibilizados em séries históricas do Instituto Brasileiro de Geografia e Estatística, o volume de recursos aplicados, os relatórios de gestão do FCO emitidos pelo Ministério da Integração Nacional e pelo Conselho Deliberativo do Fundo; o período analisado compreende informações antes da existência do fundo, proporcionando uma análise da região antes do FCO e o efeito da aplicação dez anos depois.

Para analisar a relação entre a liberação de recursos do FCO com a geração de empregos no Centro-Oeste brasileiro, realizou-se uma pesquisa descritiva, com abordagem quali-quantitativa, por meio de um estudo de caso.

O aspecto descritivo é observado na discussão dos recursos do Fundo Constitucional do Centro-Oeste, apresentando assim, uma descrição dos recursos aplicados nos municípios da amostra analisada. Em relação aos procedimentos, classifica-se como estudo de caso por não abranger os demais fundos de investimento criados e previstos na Constituição de 1988; e classifica-se como pesquisa documental por utilizar-se de informações extraídas de relatórios gerenciais históricos dos órgãos como Instituto Brasileiro de Geografia e Estatística (IBGE, 2015), Instituto de Pesquisa Econômica Aplicada (IPEADATA, 2015), formulando um banco de dados em painel para processamento dos dados.

Quanto ao método, o estudo classificou-se como quali-quantitativa, que traduz em números, informações e conclusões com o intuito de classificar, analisar e ordenar 
os resultados e qualitativa que contribui no processo de compreensão do fenômeno social segundo o comportamento dos agentes sociais em seu meio e suas atitudes.

\subsection{Dados e Modelo Teórico}

A população da pesquisa compreendeu 468 municípios da região Centro-Oeste para os anos de 1985/1996, o número de agências do banco administrador dos recursos, informações estas disponibilizadas nas séries históricas do IBGE e IPEADATA. O programa entrou em vigor em 1989, porém apenas em 1993 os dados foram medidos por institutos governamentais, e pela indisponibilidade das demais variáveis, o ano de análise do efeito do FCO foi em 1996.

Os setores analisados indústria, comércio e serviços geraram uma grande quantidade de informações, e assim foi montado um banco de dados em painel e inseridas variáveis socioeconômicas para eliminação de erros no modelo econométrico construído.

A utilização de dados regionais e nacionais torna a pesquisa de alcance macroeconômico e serão considerados dados que não podem ser observados nos relatórios de gestão emitidos pelo Ministério da Integração e CONDEL/FCO (2010), mas que estão implícitos no sistema macroeconômico como: PIB nacional, índice de inflação, índice populacional regional, o número de agências do Banco do Brasil, o número de empresas por setor. Serão consideradas essas variáveis no modelo econométrico para reduzir o efeito não observável; estes e outros fatores sociais influenciam os resultados, por isso devem ser consideradas.

O levantamento e alocação dos dados foi montada em painel, compilando séries históricas com as seguintes informações: Estados; Municípios; ano da análise das variáveis; PIB do comércio, indústria e serviços; População; Emprego do comércio, indústria e do setor de serviços; Número de empresas nos três setores; Inflação no ano de 1985 e 1996; e agências do Banco do Brasil. Assim, busca-se reduzir os efeitos não observáveis com a utilização de painel de dados sobre as variáveis macro e microeconômicas. 
As equações de processamento de dados em painel são evidenciadas abaixo, o modelo 1 trata da geração de empregos no setor de comércio, o modelo 2 para o setor da indústria e o modelo 3 para o setor de serviços:

$$
\begin{aligned}
& \text { Modelo (1): } \text { Emprego }_{\text {com }}=\beta_{0}+\beta_{1} \text { Pib }_{\text {com }_{i, t}}+\beta_{2} \text { Pop }_{i, t}+\beta_{3} F_{C O}+t+\mu_{i, t} \\
& \text { Modelo (2): Emprego } \text { ind }_{i, t}=\beta_{0}+\beta_{1} \text { Pib }_{\text {ind }_{i, t}}+\beta_{2} \text { Pop }_{i, t}+\beta_{3} F_{C O}+\mu_{i, t} \\
& \text { Modelo (3): Emprego } \mu_{\text {ser }}=\beta_{0}+\beta_{1} \text { Pib }_{\text {ser }_{i, t}}+\beta_{2} \text { Pop }_{i, t}+\beta_{3} F C O_{i, t}+\mu_{i, t}
\end{aligned}
$$

O indexador $i$ são os municípios, neste caso foram considerados todos os municípios do Centro-Oeste (468 municípios), já o indexador $t$ reflete os períodos de 1985 e 1996. Isto é, são dados antes da entrada do FCO e dados obtidos após a implantação do programa. O programa entra em vigor em 1989, porém apenas em 1993 os dados foram disponibilizados por institutos do governo, porém pela disponibilidade das demais variáveis, o ano de análise foi 1996.

Determinou-se que a variável FCO recebe valor zero (0) para os municípios que não possuem agências instaladas e valor um (1) para os municípios que tinham uma ou mais agências instaladas. Informação esta relevante a considerar, uma vez que cidades que não tem agência administradora minimiza-se a concessão dos financiamentos. $\mathrm{Na}$ criação dessa variável não foi considerado a possibilidade dos financiamentos serem aprovados nas cidades sem agências do banco administrador do fundo.

Através dos testes de hipóteses sobre o modelo econométrico, sua eficiência poderá ser utilizada para projetar ou prever valores futuros, os modelos podem ter utilização para fins de controle e políticas públicas ou na definição de metas para um objetivo que se almeja alcançar por setores.

A análise dos dados foi medida através de resultados de regressão. Nesta buscou-se entender a relação de dependência estatística entre as variáveis e análise de regressão em séries temporais estimar ou prever quanto de recurso precisa ser liberado para a criação de uma vaga de emprego. Dessa dependência observam-se alguns fatores: (a) o aumento na contratação de funcionários contribui para o aumento da população local economicamente ativa; (b) a alta no faturamento contribui na consolidação das empresas; e (c) a elevação na arrecadação de impostos contribui com a população no 
sentido que é revertido em serviços básicos para a sociedade. A sinergia é fundamental para o funcionamento entre os recursos FCO x resultados.

\section{DESCRIÇÃO E ANÁLISE DOS DADOS E RESULTADOS}

Nesta seção são evidenciadas as análises e resultados obtidos no estudo, o desmembramento dos dados do painel elaborado em planilha do Excel se faz necessário para evidenciar as observações. A primeira análise é sobre os municípios pesquisados inseridos na região Centro-Oeste; para o DF temos apenas Brasília, para o Estado de Goiás são analisados 246 municípios, para o Estado de Mato Grosso do Sul 79 e para Mato Grosso 142, totalizando 468 municípios que fazem parte da amostra pesquisada.

A seguir apresenta-se a Tabela 1 com o montante de recursos aplicados e o número de empregos estimados pelo FCO de 1990 a 2008.

Tabela 1: Recursos aplicados e número de empregos gerados

\begin{tabular}{clcclc}
\hline Ano & $\begin{array}{l}\text { Aplicações } \\
\text { FCO } \\
\text { milhões ** }\end{array}$ & $\begin{array}{l}\text { Geração de } \\
\text { empregos* }\end{array}$ & $\begin{array}{c}\text { Ano } \\
\text { Aplicações } \\
\text { FCO } \\
\text { Milhões** }\end{array}$ & $\begin{array}{c}\text { Geração de } \\
\text { empregos* }\end{array}$ \\
\hline 1990 & $\mathrm{R} \$ 313.711$ & 7.055 & 2000 & $\mathrm{R} \$ 350.800$ & 24.958 \\
1991 & $\mathrm{R} \$ 359.016$ & 14.563 & 2001 & $\mathrm{R} \$ 1.173 .886$ & 48.397 \\
1992 & $\mathrm{R} \$ 177.333$ & 10.342 & 2002 & $\mathrm{R} \$ 1.685 .008$ & 64.009 \\
1993 & $\mathrm{R} \$ 498.722$ & 24.426 & 2003 & $\mathrm{R} \$ 1.043 .494$ & 145.914 \\
1994 & $\mathrm{R} \$ 349.728$ & 23.620 & 2004 & $\mathrm{R} \$ 1.283 .463$ & 52.092 \\
1995 & $\mathrm{R} \$ 212.011$ & 16.071 & 2005 & $\mathrm{R} \$ 1.570 .656$ & 65.956 \\
1996 & $\mathrm{R} \$ 268.048$ & 17.124 & 2006 & $\mathrm{R} \$ 1.508 .941$ & 80.512 \\
1997 & $\mathrm{R} \$ 367.429$ & 21.501 & 2007 & $\mathrm{R} \$ 2.024 .620$ & 92.866 \\
1998 & $\mathrm{R} \$ 454.623$ & 31.170 & 2008 & $\mathrm{R} \$ 3.500 .283$ & 164.057 \\
1999 & $\mathrm{R} \$ 207.121$ & 23.077 & $\mathrm{TOTA}$ & $\mathrm{R} \$ 17.348 .893$ & 927.710 \\
& & & & & \\
\hline
\end{tabular}

Fonte: Adaptado das Cartilhas de programação do FCO (1990 a 2008) - Ministério da Integração. *Estimados pelo CONDEL/FCO - Elaboração própria (2015); **Valores em milhões de reais. 
O montante de recursos aplicados e o número de empregos estimados desde o lançamento do fundo na região Centro-Oeste (1990 a 2008), totalizaram $\mathrm{R} \$ 17.348$ bilhões e 927 mil vagas de empregos.

\subsection{Variável Produto Interno Bruto}

Entre os 468 municípios, os dez com maiores PIB para o comércio no ano de 1985 e 1996 estão elencados na tabela 2, formulada através dos dados disponíveis no IBGE (2015).

Tabela 2: Municípios com maior PIB - comércio

\begin{tabular}{llcllc}
\hline Sigla & Município & $\begin{array}{c}\text { Valores em } \\
\text { Reais }(1985)^{*}\end{array}$ & Sigla & Município & $\begin{array}{c}\text { Valores em } \\
\text { Reais }(1996)^{*}\end{array}$ \\
\hline GO & Goiânia & $1.413 .283,63$ & DF & Brasília & $1.380 .252,28$ \\
DF & Brasília & $1.076 .729,01$ & GO & Goiânia & $814.193,28$ \\
MS & Campo Grande & $560.889,39$ & MS & Campo Grande & $370.441,22$ \\
MT & Cuiabá & $532.032,94$ & MT & Cuiabá & $311.833,11$ \\
MS & Dourados & $344.937,91$ & GO & Anápolis & $105.563,63$ \\
GO & Anápolis & $279.926,19$ & MT & Rondonópolis & $73.177,09$ \\
MT & Rondonópolis & $202.558,95$ & MS & Dourados & $71.524,76$ \\
MT & Várzea Grande & $150.594,58$ & MT & Várzea Grande & $64.794,64$ \\
GO & Rio Verde & $147.372,96$ & GO & Rio Verde & $38.038,11$ \\
GO & Itumbiara & R $\$ 88.064,04$ & GO & Itumbiara & $36.670,51$ \\
\hline
\end{tabular}

Elaboração própria (2015)

Fonte: Dados da pesquisa - Adaptado PIB Municipal - Instituto Brasileiro de Geografia e Estatística - PIBCO - *valores atualizados ao ano 2000.

Comparando o PIB no setor do comércio antes e depois do FCO para o ano de 1985/1996 os municípios mantiveram-se os mesmos, porém se alteraram na ordem de classificação, principal mudança ocorreu com o crescimento do PIB de Brasília. Além disso, o setor teve um decréscimo de $32 \%$ na análise dos 10 maiores PIB municipais, já o decréscimo do PIB para o setor foi de $39 \%$ abrangendo todos os municípios da região Centro-Oeste.

Os dez municípios da região Centro-Oeste com o maior PIB para o setor industrial estão relacionados conforme a tabela 3: 
Tabela 3: Municípios com maior PIB adicionado - indústria

\begin{tabular}{|c|c|c|c|c|c|}
\hline$\overline{\text { Sigla }}$ & Município & $\begin{array}{l}\text { Valores em } \\
\text { Reais }(1985) *\end{array}$ & Sigla & Município & $\begin{array}{l}\text { Valores em } \\
\text { Reais } \\
(1996) *\end{array}$ \\
\hline GO & Goiânia & $236.514,82$ & DF & Brasília & $2.157 .438,40$ \\
\hline DF & Brasília & $145.275,40$ & GO & Goiânia & $1.234 .839,34$ \\
\hline MS & Campo Grande & $97.474,05$ & MS & Campo Grande & $480.438,09$ \\
\hline MT & Cuiabá & $87.390,65$ & GO & Aparecida de Goiânia & $348.769,75$ \\
\hline GO & Luziânia & $38.063,15$ & GO & Caldas Novas & $336.208,36$ \\
\hline GO & Anápolis & $33.691,89$ & MT & Cuiabá & $304.328,95$ \\
\hline MT & Várzea Grande & $24.825,73$ & GO & Senador Canedo & $200.795,00$ \\
\hline MT & Rondonópolis & $22.124,40$ & GO & Colinas do Sul & $83.154,44$ \\
\hline MS & Dourados & $17.835,97$ & GO & Cavalcante & $50.885,34$ \\
\hline GO & Itumbiara & $15.557,78$ & GO & Minaçu & $41.429,18$ \\
\hline
\end{tabular}

Fonte: Elaboração própria. Dados da pesquisa - Adaptado PIB Municipal - Instituto Brasileiro de Geografia e Estatística - PIBI - *valores atualizados ao ano 2000.

Em análise comparativa do crescimento do PIB antes e pós FCO para o setor industrial, identifica-se que em 1996 os municípios mudaram bastante, percebe-se o crescimento do setor industrial para os municípios do Estado de Goiás com os sete maiores PIB municipais do setor industrial e destaque para o setor como um todo que teve um crescimento elevado. O setor cresceu na análise dos 10 maiores PIB de 718 milhões para 5 bilhões de reais um crescimento do setor em $729 \%$.

Os dez municípios da região Centro-Oeste com o maior PIB para o setor de serviços estão elencados na tabela 4 e percebe-se tratar-se da maior atividade para a região Centro-Oeste, pois esta diretamente ligada às atividades do agronegócio.

Tabela 4: Municípios com maior PIB adicionado - serviços

\begin{tabular}{llrllr}
\hline Sigla & Município & $\begin{array}{c}\text { Valores em } \\
\text { Reais }(1985)^{*}\end{array}$ & Sigla & Município & $\begin{array}{c}\text { Valores em } \\
\text { Reais (1996)* }\end{array}$ \\
\hline DF & Brasília & $17.534 .240,89$ & DF & Brasília & $25.601 .853,26$ \\
GO & Goiânia & $3.672 .645,73$ & GO & Goiânia & $6.144 .112,13$ \\
MS & Campo Grande & $2.132 .693,79$ & MS & Campo Grande & $3.081 .294,50$ \\
MT & Cuiabá & $1.713 .376,18$ & MT & Cuiabá & $3.032 .166,53$ \\
\hline
\end{tabular}




\begin{tabular}{llllll}
\hline GO & Cachoeira de & $717.237,34$ & GO & Anápolis & $720.671,68$ \\
& Goiás & & & & \\
GO & Anápolis & $695.863,93$ & GO & Luziânia & $594.687,82$ \\
MS & Dourados & $677.000,67$ & MS & Dourados & $479.513,15$ \\
MT & Várzea Grande & $439.635,74$ & MT & Rondonópolis & $378.193,69$ \\
MT & Rondonópolis & $399.594,85$ & MT & Várzea Grande & $360.944,03$ \\
GO & Rio Verde & $290.432,82$ & GO & Aparecida de & $346.587,74$ \\
& & & & Goiânia & \\
\hline
\end{tabular}

Fonte: Elaboração própria. Dados da pesquisa - Adaptado PIB Municipal - Instituto Brasileiro de Geografia e Estatística - PIBSE - *valores atualizados ao ano 2000.

Consubstancialmente no comparativo para o setor de serviços para o período de análise antes e pós-recursos do FCO relacionam-se os dez municípios com maiores PIB 1985/1996. Para o ano de 1996 os municípios mantiveram o crescimento do PIB serviços, saltando de 28 bilhões para 40 bilhões de reais, um crescimento de $144 \%$ para o setor em 10 anos.

\subsection{Variável emprego - Setores comércio/indústria/serviços}

Em relação a variável emprego que trata do número de pessoas ocupadas para o comércio, indústria e serviços para os anos de 1985 e 1995 são evidenciadas no gráfico $1:$

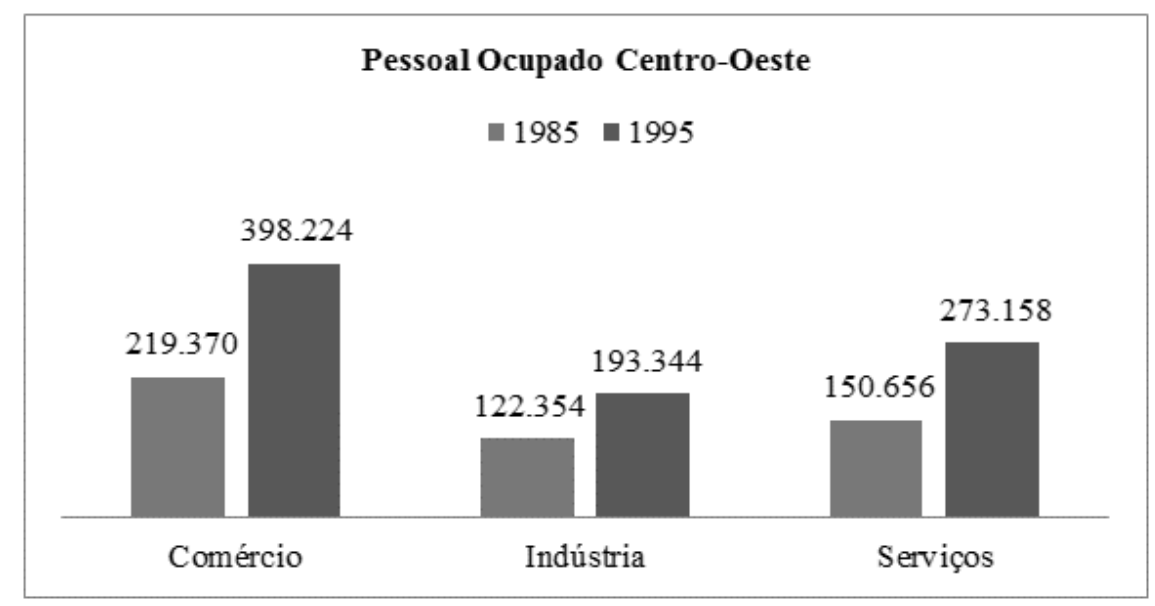

Gráfico 1: Pessoal ocupado

Elaboração própria (2015).

Fonte: Dados da pesquisa - IBGE - Instituto Brasileiro de Geografia e Estatística. EMPIN, EMPOS, EMPCO 1985/1996. 
Para todos os setores houve aumento do número de empregos gerados, para o comércio houve um crescimento de 178.854 vagas ou 48\%; Para a indústria 70.990 ou 19\% e para o setor de serviços de 122.502 ou $33 \%$ em vagas geradas.

\subsection{Variável - Abertura de empresas formais}

$\mathrm{Na}$ análise da variável empresa para os setores, que trata do número total de estabelecimentos ou unidades locais por município segundo o senso IBGE para 1985/1995. O gráfico 2 apresenta o número de estabelecimentos por setor para os anos de 1985 e 1995 :

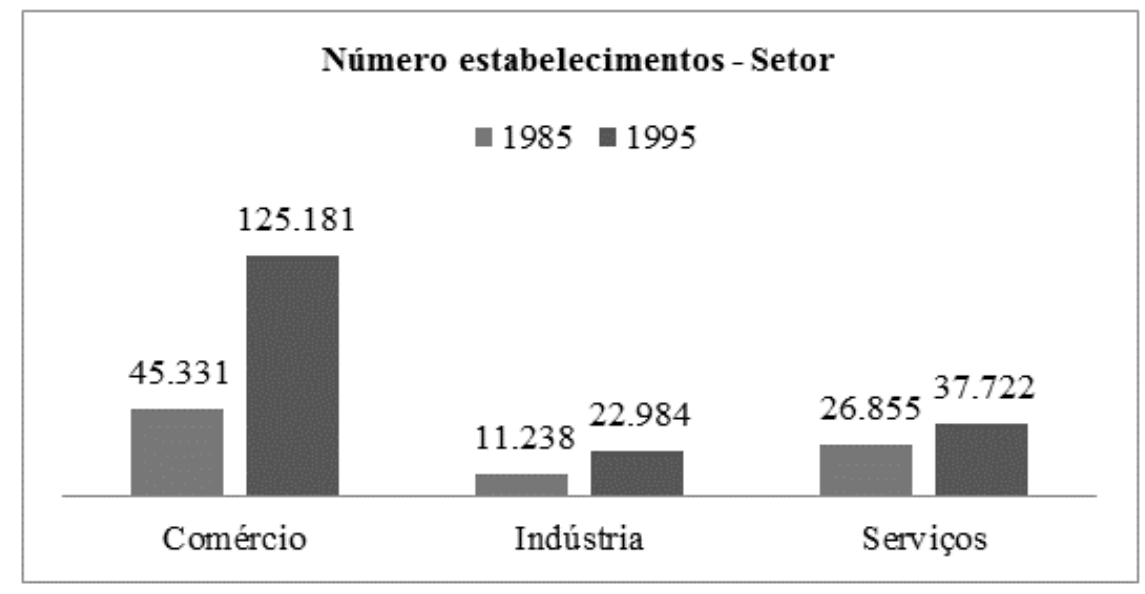

\section{Gráfico 2: Número de estabelecimentos por setor}

Elaboração própria (2015).

Fonte: Dados da pesquisa - IBGE - Instituto Brasileiro de Geografia e Estatística NECO, NEIN, NEOS.

Todos os setores apresentaram crescimento relativo ao número de empresas formais na região Centro-Oeste. No setor do comércio o crescimento de empresas foi de 79.850 ou equivalente a $78 \%$, no setor industrial o crescimento foi de 11.746 empregos ou de $11 \%$ e no setor de serviços de 10.867 novas empresas ou $11 \%$.

\subsection{Variável - Agência administradora de recursos}

$\mathrm{Na}$ análise da variável agência bancária por municípios, considerada variável para reduzir os erros ' $\mu$ ' da equação, ou seja, municípios que possuem agências 
bancárias administradoras do fundo estatisticamente utilizaram recursos do fundo, já aquelas que não possuem agências a variável é desconsiderada para o município.

Tabela 5: Número de agências do Banco do Brasil por município

\begin{tabular}{llllr}
\hline Sigla & Código & Município & Ano & Agências \\
\hline DF & 5300108 & Brasília & $\mathbf{1 9 9 6}$ & 47 \\
GO & 5208707 & Goiânia & $\mathbf{1 9 9 6}$ & 22 \\
MS & 5002704 & Campo Grande & $\mathbf{1 9 9 6}$ & 10 \\
DF & 5300108 & Brasília & $\mathbf{1 9 8 5}$ & 8 \\
MT & 5103403 & Cuiabá & $\mathbf{1 9 9 6}$ & 7 \\
GO & 5201108 & Anápolis & $\mathbf{1 9 9 6}$ & 3 \\
MT & 5108402 & Várzea Grande & $\mathbf{1 9 9 6}$ & 3 \\
GO & 5201405 & Aparecida de Goiânia & $\mathbf{1 9 9 6}$ & 2 \\
GO & 5208707 & Goiânia & $\mathbf{1 9 8 5}$ & 2 \\
GO & 5211503 & Itumbiara & $\mathbf{1 9 9 6}$ & 2 \\
GO & 5218805 & Rio Verde & $\mathbf{1 9 9 6}$ & 2 \\
MS & 5003702 & Dourados & $\mathbf{1 9 9 6}$ & 2 \\
MT & 5103502 & Diamantino & $\mathbf{1 9 9 6}$ & 2 \\
MT & 5107602 & Rondonópolis & $\mathbf{1 9 9 6}$ & 2 \\
\hline
\end{tabular}

Fonte: Elaboração própria. Dados da pesquisa - BACEN - Banco Central do Brasil Deati

A tabela 5 demonstra os municípios que possuem mais de uma agência do banco administrador dos recursos do FCO. Verifica-se que 286 municípios possuem apenas uma agência do banco administrador do Fundo e que 636 municípios desde a fundação do Banco do Brasil não tinham uma agência bancária para o período analisado. Essas informações são importantíssimas, pois desconsideram a possibilidade das empresas situadas nesses municípios sem agência receber recursos do FCO.

\subsection{Resultados de Regressão}

O resultado foi gerado através das equações apresentadas na metodologia, de acordo com o banco de dados de todas as variáveis para os anos de 1985 e 1996. Como variáveis de controle foram consideradas a população, inflação anual e agências do banco administrador 
Tabela 6: Resultado de Regressão

\begin{tabular}{lccc}
\hline VARIABLES & Modelo (1) & Modelo (2) & Modelo (3) \\
\hline Ano & $9.829 * *$ & $4.940^{* *}$ & $1.956^{* *}$ \\
& $(6.043)$ & $(4.387)$ & $(7.117)$ \\
FCO & $\mathbf{5 . 4 9 3 * * *}$ & $\mathbf{1 . 3 3 1 * * *}$ & $\mathbf{3 . 5 5 9 * * *}$ \\
& $(0.276)$ & $(0.194)$ & $(0.227)$ \\
PIB & $0.00820^{* * *}$ & $0.00865^{* * *}$ & $0.00250^{* * *}$ \\
& $(0.00105)$ & $(0.000519)$ & $(0.0000704)$ \\
População & $0.0361 * * *$ & $0.00310^{* * *}$ & $0.0169 * * *$ \\
& $(0.00112)$ & $(0.000793)$ & $(0.000908)$ \\
Constant & $-19,770^{* *}$ & $-9,751^{* *}$ & $-4,042^{* *}$ \\
& $(12,029)$ & $(8,733)$ & $(10,043)$ \\
\hline Observations & $935 * * * *$ & $930^{* * * *}$ & $934 * * * *$ \\
R-squared & 0.953 & 0.831 & 0.965 \\
\hline
\end{tabular}

Fonte: Elaboração própria.

Standard errors in parentheses

$* * * \mathrm{p}<0.01, * * \mathrm{p}<0.05, * \mathrm{p}<0.1$

$* * * *$ missing values disregarded

Na regressão, a constant apresenta a metodologia da diferença de médias: para chegar nesses resultados, foram feitas as regressões para 1985, considerando FCO =0, e novas regressões para $1996 \mathrm{FCO}=1$. Com isso, todas as variáveis que são fixas no tempo, as não observáveis, inclusive, são canceladas nesse método, restando apenas os efeitos da variável de tratamento. Sendo assim, para o comércio o FCO gerou uma média de 5.493 empregos, já para a indústria teve um aumento em média de 1.331 empregos e para os serviços 3.559 empregos; todos os resultados foram estatisticamente significantes.

Para o controle considerou-se o PIB de cada município, uma vez que, com o crescimento municipal isso reflete diretamente no nível de emprego. Assim, como a população também foi controlada, quanto mais populoso for o município, mais empregos diretos são observados. Diante dessas variáveis controladas, apresenta-se com certa robustez o efeito do FCO para os municípios.

\section{Considerações Finais}

A realização desta pesquisa se desenvolveu por questionar a relação entre os recursos liberados pelo FCO e a geração de empregos, buscando verificar se o fundo cumpre com seu objetivo. De modo geral, a análise desta pesquisa mostra que os 
recursos do FCO influenciam na geração de empregos. Os resultados atendem as deficiências levantadas na justificativa da pesquisa, pois apresenta avaliação e estudo da política regional, tornando possível verificar a eficácia da aplicação do dinheiro público, conforme mencionado por Bresser (2008) em análise da eficácia das políticas adotadas para o desenvolvimento de um Estado-nação.

Conforme objetivo proposto de analisar a relação entre a aplicação dos recursos do FCO e a geração de empregos, além de demonstrar a importância do fundo, foi necessária a construção de um banco de dados em painel completo e coeso. Através da regressão deste painel tornou-se possível a avaliação do efeito do FCO na geração de empregos para os anos observados (1985 e 1996).

O primeiro objetivo específico em demonstrar a importância do FCO para a região Centro-Oeste foi atendido ao realizar a análise que identificou o crescimento da economia regional e setorial avaliado pelo PIB dos setores. O setor industrial elevou seu índice em 729\%; o setor de serviços em 144\%; e o PIB do comércio apresentou decréscimo em $32 \%$, porém apresentando elevação no percentual de empresas abertas em $48 \%$.

Em consonância com este objetivo conclui-se que os números de empresas formais em todos os setores apresentaram crescimento. No setor do comércio o crescimento de empresas foi de $78 \%$; no setor industrial o crescimento foi de $11 \%$ e no setor de serviços de $11 \%$. Referente à geração de empregos o setor de comércio elevou aproximadamente $50 \%$ das vagas, o setor industrial $19 \%$ e o setor de serviços $33 \%$. Em razão do objetivo da pesquisa em verificar o efeito do FCO na geração de empregos conclui-se que do crescimento do número de empregos para os setores analisados (comércio, indústria e serviços) o FCO apresentou efeito significativo, notado pelo resultado de regressão.

Dentre as principais contribuições que o objetivo específico construir um modelo econométrico apresentou foi gerar o resultado de regressão. Salienta-se entre os resultados para o comércio que a média foi de 5.493 empregos gerados, para o setor industrial média 1.331 empregos e para o setor de serviços a média foi de 3.559 vagas, totalizando 10.383 vagas nos setores analisados. Conclui-se que este total é considerado relevante frente ao total de empregos gerados no período em todos os setores. A média de regressão equivale a $28 \%$ das vagas geradas no período para todos os setores da 
região, bem como o resultado de regressão alcança o equivalente a $65 \%$ das vagas estimadas pelo programa na liberação dos investimentos.

Duas hipóteses foram testadas, H0 a qual acreditava que a liberação de recursos do fundo não influenciava o número de empregos gerados, sendo esta rejeitada e H1 que existia relação entre a aplicação dos recursos do FCO e o número de empregos gerados, sendo aceita, visto que os resultados são significantes estatisticamente através do teste de regressão. Sugere-se como melhoria deste trabalho utilizar a mesma equação para os anos 2000, 2005, 2010, 2015 e nos demais setores econômicos.

\section{Referências}

ALCOFORADO, Fernando. Os condicionantes do desenvolvimento do Estado da Bahia. Barcelona, 2003. Dissertação (Doutorado em Planificacíon Territorial y Desarrollo Regional) Tradução: Ordenamento do Território e do Desenvolvimento Regional. Universitat de Barcelona, 2003.

BANCO DO BRASIL, Roteiro para elaboração de projeto de investimento: Roteiro A - Empresarial, 2015.

BANCO MUNDIAL. Brazil: public spending on social programs: issues and options, v. I, maio, 1988.

BEVILACQUA, Lucas. Intervenção do Estado na agricultura: política de desenvolvimento agrário, tributação e incentivos fiscais. 2010.

BRASIL, Constituição Federal, 1988.

Cartilha FCO, Conselho Deliberativo do Fundo Constitucional do CentroOeste, Ministério da Integração, Banco do Brasil S/A. 2001.

, Programação FCO, Conselho Deliberativo do Fundo Constitucional do Centro-Oeste, Ministério da Integração, Banco do Brasil S/A. 2001.

fiscais. , Lei $\mathrm{n}^{\mathrm{o}} 7.827$, de 27 de setembro de 1989. Fundos regionais e incentivos , Ministério da Integração Nacional. Fundo Constitucional do CentroOeste. Disponível em: http://www.integracao.gov.br/apresentacao14. Acesso 05/03/2012.

Ministério da Integração Nacional. Fundos Constitucionais de Financiamento 20 anos: Desempenho Operacional no exercício de 1989/2008. Brasília. 2010. 
BRESSER, Luiz C. Pereira. O modelo estrutural da gerência pública. Revista de Administração Pública 42 (2): 391-410, mar/abr. 2008. Editora FGV. Rio de Janeiro. 2008.

BRUM, Argemiro Jacob. O Desenvolvimento Econômico Brasileiro. Ijuí - RS: Vozes, 4. ed. 1984.

BUARQUE, Cristovam. Avaliação Econômica de Projetos. Rio de Janeiro: Campus, 1991.

COLON, Leandro. Brasil tem $3^{\mathbf{0}}$ pior índice de desigualdade no mundo. 2010. Disponível em: http://www.estadao.com.br/noticias/geral,brasil-tem-3-pior-indice-dedesigualdade-no-mundo,585341,0.htm Acesso em 20/01/2013.

FALCINI, P. Avaliação econômica de empresas - técnica e prática. 2. ${ }^{a}$ ed. São Paulo: Atlas, 1995.

DATASUS, 1981-1990, 1992-1999, 2001-2006: IBGE - Estimativas preliminares para os anos intercensitários dos totais populacionais, estratificadas por idade e sexo pelo MS/SGEP/Datasus. Acesso em outubro/2014.

IBGE, Diretoria de Pesquisas, Departamento de Contas Nacionais. Contas Regionais do Brasil 1985-2000. Disponível em: http://www.ibge.gov.br/ home/estatistica/economia/contasregionais/ Acesso em 09/03/2015.

IPEADATA, PIB Municipal - comércio/indústria/serviços - valor adicionado preços básicos - R\$, a preços do ano 2000 (mil) - Instituto Brasileiro de Geografia e Estatística - PIBCO. Disponível em: http://www.ipeadata.gov.br/ Acesso em $11 / 03 / 2015$.

População residente - $\mathbf{1}^{\circ}$ de julho - estimativa - Habitante - Instituto Brasileiro de Geografia e Estatística - ESTIMA_PO. http://www.ipeadata.gov.br/ Acesso em 11/10/2015.

, Número total de Estabelecimentos ou Unidades Locais Comércio/indústria/serviços - Unidade - Instituto Brasileiro de Geografia e Estatística - NECO. http://www.ipeadata.gov.br/ Acesso em 11/10/2014.

Instituto Brasileiro de Geografia e Estatística, Sistema Nacional de Índices de Preços ao Consumidor (IBGE/SNIPC). http://www.ipeadata.gov.br/ Acesso em $12 / 10 / 2015$.

Pessoal ocupado - Indústria/serviços/comércio - Pessoa - Instituto Brasileiro de Geografia e Estatística - EMPIN. http://www.ipeadata.gov.br/Acesso em $12 / 10 / 2015$.

LAMPREIA, Luiz Felipe. Relatório brasileiro sobre desenvolvimento social. Estudos avançados, Centro de Documentação e Pesquisa Vergueiro, 1995.

LUNA, Francisco Vidal; KLEIN, Hebert S. Desigualdade e indicadores sociais no Brasil. Rio de Janeiro. Editora FGV, 2009. P. 97-116.ISBN 978-85-225-0736-8. 
MONTEIRO NETO, Aristides; GOMES, Gustavo Maia; Quatro Décadas de Crescimento Econômico no Centro-Oeste Brasileiro: Recursos Públicos em Ação. Publicado na revista Econômica do Nordeste, Fortaleza, v. 30, n. Especial 856-875, dezembro 1999.

MORENO, Gislaene; HIGA, Tereza Cristina Souza. Geografia de Mato Grosso: território, sociedade, ambiente. Cuiabá-MT: Entrelinhas, 2005.

OLIVEIRA, Héder Carlos. Desigualdade regional e os fundos constitucionais de financiamento no Brasil. Belo Horizonte - MG, 2005. Dissertação (Mestrado em Economia) - Programa de Pós-Graduação em Economia, PPGE/UFMG, 2005.

PEREIRA, Filipe Keuper Rodrigues; PÔRTO JÚNIOR, Sabino da Silva. Desigualdade regional de renda no Brasil - análise e previsão. Estudos do Cepe, Santa Cruz do Sul -RS, v. 14, p. 29-52, 2001.

ROSS, Stephen A.; WESTERFIELD, Randolph W.; JAFFE, Jeffrey F. Administração Financeira: Corporate Finance. 2. ed. São Paulo: Atlas, 2002.

ROLLEMBERG, Rodrigo; Parecer da comissão de meio ambiente, defesa do consumidor e fiscalização e controle (CMA) - processo de prestação de contas do Fundo Constitucional de Financiamento do Centro-Oeste (FCO) relativo ao exercício de 2011, e cópia do parecer conjunto elaborado pelo Ministério da Integração Nacional e a Superintendência de Desenvolvimento do Centro-Oeste (SUDECO). Senado Federal; 2013.

SILVA, José A. Comentário Contextual à Constituição. Malheiros. São Paulo. 2005.

World Trade Organization. World Trade Report: Exploring the links between subsidies, trade and the WTO. 2006. Tradução: Organização Mundial do Comércio (OMC) - Relatório Mundial do Comércio: Explorando as ligações entre os subsídios, o comércio e o WTO. 2006.

Submetido em: 11/02/16

Aceito em: 04/05/16 